\title{
Original Article: Study of Thyroid Dysfunction in Diabetic Patients
}

\author{
Abhay Tirkey (MD) ${ }^{1}$, Devendra Ahirwar (MD) ${ }^{2}$, Kiran Tandia (MD) ${ }^{3}$ \\ ${ }^{1,2}$ Assistant Professor, Department of Medicine Bundelkhand Medical College Sagar \\ ${ }^{3}$ Senior Resident, Department of Medicine Bundelkhand Medical College Sagar
}

\begin{abstract}
Introduction: Diabetes is a modern pandemic. Any disorder that is even weakly correlated to it deserves special attention. Thyroid disorders are second MC endocrine dysfunctions. As comorbid condition these together throw a great burden to medicine and humanity. Considering the ever increasing population of diabetics in our country and the significant causal relationship established by current literature, this study was undertaken. Aims and Objectives: To assess the prevalence of different forms of thyroid disorders viz. hypothyroidism, hyperthyroidism, subclinical hypothyroidism and subclinical hyperthyroidism among diabetic patients. To compare the prevalence of various thyroid disorders between T1DM AND T2DM. Material and Methods: 100 patients admitted/out patients who were known cases or recently diagnosed with Diabetes Mellitus(DM) fulfilling the inclusion and exclusion criteria and giving informed consent were included in the study. Fasting TSH, T3, T4, FT3, FT4 and recent blood sugar levels measured along with other routine laboratory investigations. TSH, T3, T4, FT3, FT4 were estimated by chemiluminescence method. Results: Of the 100 patient studied 21 were suffering from Type I DM whereas the rest had Type II DM. The prevalence of euthyroid, hypothyroid, hyperthyroid, subclinical hypothyroid \& subclinical hyperthyroid was $76 \%, 12 \%, 3 \%, 5 \%$, 4\% respectively. Discussion: the study concluded that the incidence increased with duration of diabetes which was clinically and statistically significant.
\end{abstract}

Keywords: Diabetes mellitus, Thyroid dysfunction, chemiluminescence method, Fasting Blood sugar, Fasting TSH.

\section{Introduction}

Diabetes Mellitus (DM) is one of the most common chronic medical diseases worldwide with an estimated prevalence of detected DM being 3 to $4 \%$ in the general population. ${ }^{1}$ It is estimated to affect 380 million people by the year $2025^{2}$. It is one of those diseases that cannot be cured, but can only be controlled so as to prevent its long term microvascular and macrovascular complications.

Diseases of the thyroid gland are also amongst the most abundant endocrine disorders in the world second only to diabetes $^{3}$. Hyperthyroidism and hypothyroidism occur in about $2 \%$ and $1 \%$ of the population respectively ${ }^{4}$. However the endemic zones, goiter due to dietary iodine deficiency can occur in upto $15 \%$ of the population ${ }^{5}$. Most reports estimate that the prevalence of thyroid dysfunctions in this part of the world is higher in values elsewhere in the world. Many attribute it to the iodine deficient diet and others to the genetic makeup of the population. ${ }^{6}$

The researchers world over have found a higher incidence of thyroid dysfunctions among the diabetic patients. Although the prevalence is much more in case of Type I DM, a significant prevalence is also found in type II DM population, which is far higher than that found in the general population. However, the figures that have been published by various researchers tend to vary. It has been reported that up to $13.4 \%$ of patients have thyroid dysfunctions with the highest prevalence in patients with type I DM as compared to Type II DM $(6.8 \%)^{7}$. Studies in India have shown that 28$30 \%$ of patients with $\mathrm{T} 2 \mathrm{DM}$ have thyroid dysfunctions, mainly in the form of clinical or subclinical hypothyroidism ${ }^{8}$.
One of the consistent findings among various studies on metabolic disorders has been simultaneous multi glandular endocrine dysfunction. As Diabetes is a modern pandemic any disorder that is even weakly correlated to it deserves special attention. In addition to the autoimmune link between T1DM and thyroid diseases \& the increased prevalence of thyroid dysfunctions in obese individuals, the prevalence of both diabetes \& Thyroid disorders are found to increase with age, hence, further contributing to high association between the two diseases. According to some experts, thyroid dysfunctions should be evaluated yearly in all patients with diabetes ${ }^{9}$.

Although screening for thyroid dysfunctions in diabetic patients at initial diagnosis may identify a significant pool of previously undiagnosed thyroid disease ${ }^{9}$, the yield from annual screening which has been recommended by some authors ${ }^{10}$, had not been evaluated in large outpatient diabetics till 1995 by Perros et $\mathrm{al}^{11}$. Considering the ever increasing population of diabetics in our country and the significant causal relationship established by current literature, this study was undertaken.

\section{Aims and Objectives}

- To assess the prevalence of different forms of thyroid disorders viz. hypothyroidism, hyperthyroidism, subclinical hypothyroidism and subclinical hyperthyroidism among diabetic population

- To compare the prevalence of various thyroid disorders between T1DM AND T2DM

\section{Inclusion Criteria}

1) Patients admitted in medical wards and attending medical OPDs of GMC, Bhopal who were known cases or 


\section{International Journal of Science and Research (IJSR) \\ ISSN (Online): 2319-7064}

Index Copernicus Value (2013): 6.14 | Impact Factor (2014): 5.611

recently diagnosed with Diabetes Mellitus(DM)ype I \& II.

2) In case of recent onset diabetes, at least three fasting blood sugar levels above normal values were taken and $\mathrm{HbA1C}$ assay were done to establish the diagnosis of diabetes.

3) Patients who gave informed consent

\section{Exclusion Criteria}

1) Patients of drug induced hypoglycemia. Eg. High dose steroid, pentamidine and diazoxide.

2) Patients who undergone thyroid surgery \& radio iodine $\&$ other radiation exposure in head and neck region.

3) Patient not willing to participate in the study.

\section{Material and Methods}

Sample Size: 100. It was observational cross-sectional study done in Department of Medicine of Bundelkhand Medical College Sagar.

Total 100 patients both male and female aged between 1580 years were included in this study.

All patients were subjected to a designated questionnaire \& detailed physical examination. The TSH, T3, T4, FT3, FT4 and recent blood sugar levels measured along with other routine laboratory investigations, like complete blood counts, Blood Urea Levels and serum creatinine, Urine analysis, Chest $\mathrm{X}$ ray and ECG.

Fasting blood samples was taken for thyroid function and TSH, T3, T4, FT3, FT4 were estimated by chemiluminescence method. The reference normal range using this method was:

TSH : $0.3-5.5 \mu \mathrm{IU} / \mathrm{ml}$

T3 :60-200 ng/dl

$\mathrm{T} 4: 4.5-12.0 \mu \mathrm{g} / \mathrm{dl}$

FT3 : $2.4-4.2 \mathrm{pg} / \mathrm{ml}$

FT4 :0.8-1.17ng/dl

\section{Results and Discussion}

A total of 100 patients with Diabetes Mellitus were studied and as seen in Table 1, the prevalence of thyroid dysfunction among them was $24 \%$ which is far higher than previous studies evaluating this.In Type I DM pt the prevalence was $19.04 \%$ (p value :0.263) while in Type 2 DM patients it was $25.31 \%$ ( p value :0.9679).

Table 2 show the sex distribution of thyroid dysfunction profile in diabetics. It can be easily inferred that all forms of thyroid diseases are more prevalent in female population. The prevalence of thyroid dysfunction was significantly higher in Type II DM a.c.t. Type I DM.

Primary hypothyroidism as well as hyperthyroidism is equally prevalent in T1DM while in T2DM pts. Primary hypothyroidism was almost ten times more prevalent than primary hyperthyroidism
In both type I and type II DM, subclinical thyroid dysfunction outweighs overt thyroid abnormalities thereby highlighting the need of clinical screening as routine. Subclinical hypothyroidism is the clinical entity with maximal prevalence in diabetics.

There is a clear sex preponderance with female diabetics bearing the burnt of thyroid dysfunction. Although there is a clustering of cases in the age group 40-70 yrs, the increasing prevalence of thyroid dysfunction with age and duration of diabetes mandates that annual screening strategy has a far greater role to play than mere initial screening at the diagnosis of diabetes.

\section{Conclusion and Implications}

The study concluded that thyroid dysfunction increases with duration of diabetes thereby underlining the importance of annual screening of thyroid function in diabetics over and above the initial screening at the time of diagnosis. Identification and effective treatment of thyroid dysfunction is thus an essential component of high quality clinical care of patients with chronicmedical illness like DM in the specialty medical setting

\section{Acknowledgement}

We sincerely thank to all members of Department of Medicine of Bundelkhand Medical College Sagar.

\section{References}

[1] Amos A, Mcarthy D. The Rising global burden of diabetes and itscomplications: Estimates and Projections to the year 2010. Diabetic Medicine 1997;14:S7-85.

[2] Powers A C. Diabetes mellitus. Harrison's Principles of internal Medicine 18thed. McGrawHill Pub 2012.29682997.

[3] 3., 4., 5. Heuck $C$ C, Kallner $A$ et al. Diagnosis and monitoring of diseases of thyroid. WHO 2000

[4] Rathcliffe G E, Lowry A, Mashiter G et al. Thyroid Hormone concentrations in Nepal: a study of potential Gurkha army recruits. The effect of changes $n$ diet. J R Army Med Corps 1991 Feb :137(1) 14-21.

[5] Perros P, McCrimmon $R$ J, Shaw $G$, Frier $B$ M. Frequency of thyroid dysfunction in diabetic patients: value of annual screening. Diabet Med1995, 12(7):622627.

[6] Kiran Babu Atul Kakar S P Byotra. Prevalence of thyroid disorder in type II DM patients. J Assoc Phys Ind $2001: 49,43$

[7] Simme Dube, ritu Nigam, Susma Ikka. Evaluation of thyroid function in Non insulin dependent diabetes mellitus.J Assoc Physician Ind, 2001:49, 196

[8] American Diabetes Association. Screening for diabetes. Diabetes Care 2002;25(1):512-24

[9] McKenna M J, Herskowitz R, Wolfsdorf J I. Screening for thyroid diseases in children with IDDM.Diabetes Care, 1990: 13: 801-803 
International Journal of Science and Research (IJSR)

ISSN (Online): 2319-7064

Index Copernicus Value (2013): 6.14 | Impact Factor (2014): 5.611

Table 1: The prevalence of thyroid dysfunction in diabetics

\begin{tabular}{|c|c|c|c|c|c|}
\hline Diabetes Type & Euthyroid & Hypothyroid & Hyperthyroid & Subclinical Hypothyroid & $\begin{array}{c}\text { Subclinical } \\
\text { Hyperthyroid }\end{array}$ \\
\hline TYPE I DM & 17 & 2 & 2 & - & - \\
\hline TYPE II DM & 59 & 10 & 1 & 5 & 4 \\
\hline
\end{tabular}

Table 2: The sex-wise distribution of prevalence of thyroid dysfunction in diabetics

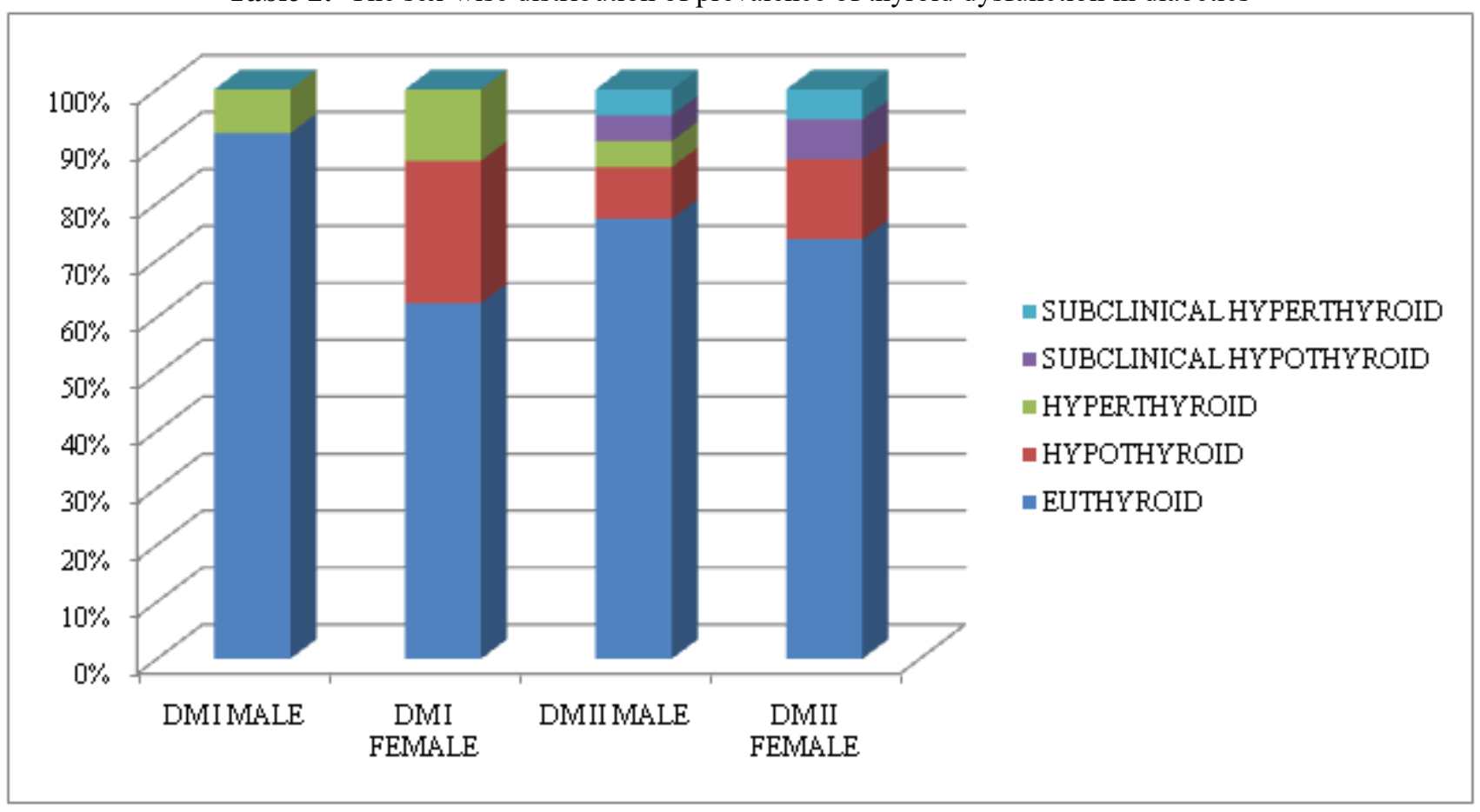

Table 3: Association of duration of diabetes with thyroid dysfunction in diabetics

| 5 - 10 Year

Volume 4 Issue 11, November 2015 